\title{
Critically Ill Pediatric Oncology Patients: What the Intensivist Needs to Know? Pediatric Critical Care Medicine
}

\author{
Amin Al Haj Moussa ${ }^{1}$, Ata Ur Rehman Maaz ${ }^{2}$, Nesreen Faqih ${ }^{3}$, Manu Sundaram ${ }^{4}$
}

\begin{abstract}
Cancer is an evolving cause of morbidity and mortality in children worldwide. In recent decades, there has been a significant increase in the survival of children with cancer, after applying new methods and treatment protocols in practice. However, the complexity of the disease itself, as well as the intensity and toxicity of treatment is such that many children require admission to the pediatric intensive care unit (PICU) which should be well equipped and led by personnel who have adequate training and expertise to provide optimum care to these complex patients. Most oncology patients who require PICU admission categorized into oncological emergencies, and/or decompensation from treatment and its side effects. In this study, we provide a summary of the essential and most recent evidence-based recommendations from published reviews and articles to aid PICU physicians and to ensure the best treatment and outcome possible for the children with such disease.

Keywords: Cancer, Critical care, Intensive care, Oncology, Pediatrics.

Indian Journal of Critical Care Medicine (2020): 10.5005/jp-journals-10071-23693
\end{abstract}

\section{BACKGROUND}

The huge improvement in the survival rate of oncology patients in the last decade is a result of a better understanding of the disease. Although the overall outlook of pediatric cancer is excellent according to recent data, this success has not been universal due to multiple issues like the lack of medical or nursing expertise in this field, limited access to anticancer treatments, and absence of concepts such as multidisciplinary management.

Therefore, more specialized and trained personal with focus on oncological problems is warranted to achieve better outcomes. ${ }^{1-4}$ On the contrary, the reported statistics showed the need for pediatric intensive care unit (PICU) admission for one in three children with cancer during their illness, making this disease one of the common causes of admission to PICU. ${ }^{5-9}$

Cancer can affect any system of the human body and can compromise its vital functions which often requiring advanced life support aids available in PICU.

Additionally, some cancers either inherently or because of therapy behave like chronic diseases with periods of decompensation which need intensive care treatment such as congestive heart failure, chronic respiratory failure, and encephalopathies.

\section{What Intensivist Needs to Know?}

The specificity of such patient and their unique presentation require from the intensivist to know the following:

- The type of oncology illness and its stage.

- The degree of disease severity, the distribution, and/or metastasis if present.

- The availability of oncology service in the hospital as support side by side with the intensivist.

- The approved policies and regulations for the treatment.

- The expected complications from treatment or palliative care measures if needed.

- Oncology emergencies and life-threatening complications.

- The availability of equipment's and other subspecialties if needed.
1,3,4 Department of PICU, Sidra Medicine, Doha, Qatar

${ }^{2}$ Department of Hematology and Oncology, Sidra Medicine, Doha, Qatar

Corresponding Author: Amin Al Haj Moussa, Department of PICU, Sidra Medicine, Doha, Qatar, Phone: +97470077630, e-mail: dr_ amin1986@yahoo.com

How to cite this article: Al Haj Moussa A, Maaz AUR, Faqih N, Sundaram M. Critically III Pediatric Oncology Patients: What the Intensivist Needs to Know? Pediatric Critical Care Medicine. Indian J Crit Care Med 2020;24(12):1256-1263.

Source of support: Nil

Conflict of interest: None

Indications for PICU admission can be broadly categorized into:

- Oncological emergencies.

- Decompensation from treatment and its side effects.

In reported statistics, respiratory failure accounts for $45 \%$ of all oncology admissions to PICU, sepsis and septic shock $30 \%$, neurological problems 15\%, while tumor lysis syndrome (TLS) and renal dysfunction account for $10 \%^{8-12}$ (Fig. 1).

\section{Oncology Emergencies}

Hyperleukocytosis

Hyperleukocytosis refers to high white blood cell count, that is, greater than $50 \times 10^{9} / \mathrm{L}(50,000 / \mu \mathrm{L})$. This abnormal lab value commonly observed in patients with acute leukemia. The reflective measurement of the tumor burden can be used in clinical practice as anticipation of complications such as hyperviscosity syndrome and risk of thromboembolism. This is crucial information for the bedside physician which can be used in decision-making in regards to approach modalities such as how much hydration fluids will be required to avoid hyperviscosity syndrome, at the same time not to induce heart failure from fluid overload, and how fast and intensive the induction chemotherapy should be. 


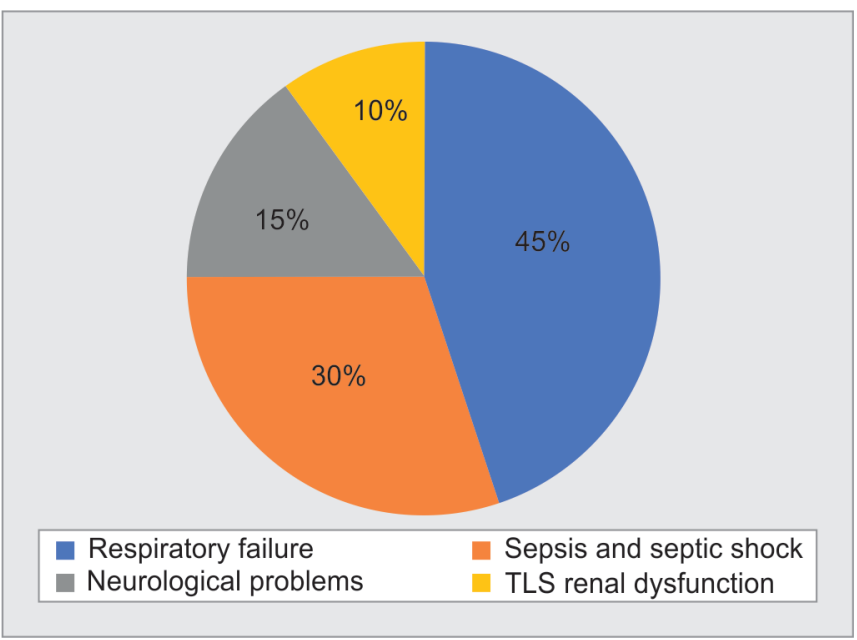

Fig. 1: Causes of PICU admission for oncology patients

Leukostasis (symptomatic hyperleukocytosis) is a medical emergency happens when white blood cell plugs occlude the small blood capillaries reducing blood flow to the tissues and results in excessive production of inflammatory cytokines due to tissues hypoxia. $^{13,14}$

These cytokines can result in endothelial damage and subsequent microhemorrhages worsen tissue hypoxia. Furthermore, these plugs can affect the lungs in the form of increase work of breathing and desaturation due to ventilation and perfusion mismatch with or without interstitial lung infiltrates. Central nervous system (CNS) can be affected too in the form of decrease visual acuity, headache, dizziness, gait instability, confusion, and in severe cases coma. In addition to that patients who present with hyperleukocytosis have an increased risk of intracranial hemorrhage perhaps from a reperfusion injury.

Brain imaging with noncontrasted computed tomography (CT) or magnetic resonance imaging (MRI) is indicated in patients with neurologic abnormalities. Clinicians must be cautious about using intravenous contrast dye when renal function may be compromised by leukostasis.

Treatment and recommendations: Treatment should prevent further plug formation and should be directed toward the rapid establishment of adequate tissue perfusion. Hyperhydration is very important to avoid hyperviscosity, using cytoreduction chemotherapy such as hydroxyurea can be indicated in cases refractory to hyperhydration or in cases of heart failure from hyperhydration. Leukapheresis can be considered as an option in patients who are refractory to treatment with no clear superiority over cytoreductive chemotherapy in terms of survival. ${ }^{15}$

\section{Superior Vena Cava Syndrome}

Superior vena cava syndrome (SVCS) refers to the clinical findings that appear on the patient in result to compression or obstruction of the SVCS.

The most important reason of SVCS is the compression induced by tumor mass such as nearby lymphoma, neuroblastoma, or a germ-cell tumor. Other causes such as a veno-occlusive thrombus as a paraneoplastic phenomenon or related to the central venous access device. SVCS resulting from a tumor mass typically presents at the time of diagnosis or relapse, while occlusive thrombus can lead to SVCS at any time during treatment.
Presenting features include cough, shortness of breath, wheezy chest, chest pain, facial swelling, headache, orthopnea, hoarseness, and blood pressure changes.

The diagnosis usually achieved by chest X-ray or CT scan demonstrating obstruction or narrowing of SVCS either by a venous thrombus or by a tumor mass, alongside secondary features of pleural and pericardial effusions, pulmonary edema, and cardiomegaly.

Treatment and recommendations: Supportive measures include optimal positioning of the child to minimize the obstruction, oxygen inhalation, and rarely mechanical ventilation. Endotracheal intubation in this circumstance can be extremely challenging and requires the most experienced physician.

Every effort should be made to establish the tissue diagnosis by means other than biopsies of the mediastinal mass, such as flow cytometry of peripheral blood/bone marrow aspirate, an assay of tumor markers such as alfa fetoprotein, or beta subunit of human chorionic gonadotropin, urinary estimation of urinary vinyl mandelic acid and homovanillic acid, and pleural or pericardial fluid cytology. However, SVCS is a true oncological emergency that by itself justifies starting chemotherapy even before confirmation of the histopathological diagnosis. ${ }^{16}$

\section{Spinal Cord Compression}

Spinal cord compression results from a tumor mass invading the vertebrae leading to their collapse resulting in compression of nerve roots. Alternatively, increased pressure in the spinal canal causes cord edema, venous hemorrhage, and ischemia.

Among the most common causes of spinal cord compression are neuroblastoma, non-Hodgkin lymphoma, and sarcoma. Bony vertebral metastatic lesions can also result in spinal cord compression in some patients. Although it occurs less frequently in pediatric practice, clinical symptoms depend on the spinal involvement, almost all sharing back pain, relatively symmetrical motor weakness, gait abnormalities, bowel, bladder dysfunction, and numbness. Diagnosis is based on a complete neurologic examination and typical features on MRI. ${ }^{17,18}$

Treatment and recommendations: Treatment can include the use of high-dose steroids and urgent chemotherapy, if the primary diagnosis is known.

The most immediate and effective treatment is neurosurgical decompression of the cord via laminectomies of the most affected vertebrae, as it leads to immediate relief of paraplegia in addition to providing access to all important tissues for diagnosis.

\section{Tumor Lysis Syndrome}

The TLS is a potentially life-threatening condition for children with blood cancers and minority of solid tumors with large tumor burden either due to cytotoxic therapy or spontaneously. Various definitions of TLS have been identified, which mainly describe the severity of renal compromise.

Massive tumor cell turns over and cell lysis results in the rapid release of potassium, phosphorus, and nucleic acids, which are metabolized into hypoxanthine, then xanthine, and finally uric acid. Hyperkalemia can cause cardiac arrhythmias. Hyperphosphatemia can cause secondary hypocalcemia, leading to neuromuscular tetany, and seizure. Uric acid can induce acute kidney injury not only by intratubular crystallization but also by crystal-independent mechanisms, such as renal vasoconstriction, 
impaired autoregulation, decreased renal blood flow, oxidation, and inflammation. ${ }^{19}$

Presenting features include electrolyte's imbalances, high potassium, phosphorus, magnesium, and uric acids in the plasma, which can lead to uric acid crystallization in kidneys tubules lead to acute renal failure.

Diagnosis mainly achieved by strict follow-up labs for electrolytes, lactate dehydrogenase, and uric acid level every 4-6 hours.

Treatment and recommendations: The goal of effective TLS management is the maintenance or restoration of normal kidney function with the rapid elimination of tumor lysis products. ${ }^{20}$ There are lots of treatment protocols for TLS but in general, it shares the same principles as shown in Table 1.

\section{Decompensation from Treatment and Its Side Effects}

\section{Cancer and Respiratory System}

Some children present with overwhelming lung infections with bacterial or fungal pathogens resulting in respiratory failure. If severe pulmonary involvement observed, the patient commonly requires mechanical ventilation.

The final pathway for such patients is the development of acute respiratory distress syndrome (ARDS), which is defined by established and agreed worldwide clinical and parameters included criteria. ${ }^{26,27}$

Noninvasive ventilation (NIV) is the preferred method of support for these patients as they are highly susceptible to infections and their mucosa membranes are fragile, increasing the risk of mucositis.

Table 1: Summary of tumor lysis syndrome metabolic abnormalities and its treatment ${ }^{21}$

\begin{tabular}{lll}
\hline Clinical problem & Approach & Dosages \\
\hline Renal insufficiency and & IV fluids dialysis & $0.9 \%$ sodium chloride at $3 \mathrm{~L} / \mathrm{m}^{2} /$ day $($ Or 200 \\
hypovolemia & & $\mathrm{mL} / \mathrm{kg} /$ day if the child is $\leq 10 \mathrm{~kg})$.
\end{tabular}

Comments and recommendations

- Use with caution in cases of kidney failure

- Monitoring of serum sodium concentration (along with tumor lysis laboratory monitoring) is recommended to avoid hyper/ hyponatremia, especially in the setting of impaired urine output

- If requiring more than $3 \mathrm{~L} / \mathrm{m}^{2} /$ day IV hydration, the urine output should be approximately $100 \%$ of total fluid intake. Consider dialysis and expert consult

Hyperuricemia Allopurinol Children $<6$ years: $150 \mathrm{mg}$ daily

- Reduce dose in renal failure; multiple drug interactions (6-mercaptopurine and azathioprine)

- IV allopurinol should be used only in patients unable to take oral medications

Children $>10$ years and Adolescents: 600 to $800 \mathrm{mg}$ daily for $2-3$ days in 2-3 divided doses Weight-directed dosing:

$10 \mathrm{mg} / \mathrm{kg} /$ day divided every 8 hours; maximum daily dose: $800 \mathrm{mg} /$ day

BSA-directed dosing:

50-100 mg/m²/dose every 8 hours; maximum daily dose: $300 \mathrm{mg} / \mathrm{m}^{2} /$ day

IV: (BSA-directed dosing):

Children and adolescents:

Initial: $200 \mathrm{mg} / \mathrm{m}^{2} /$ day administered once daily or in equally divided doses at 6-, 8-, or 12-hour intervals

Rasburicase $\quad 0.05-0.2 \mathrm{mg} / \mathrm{kg}$ IV once daily up to 5 days

Hyperphosphatemia Sevelamer Children $\geq 6$ years and adolescents:

- Contraindicated in G6PD deficiency risk of sensitization and allergic reactions

- Low phosphorus diet

Oral: $\mathrm{BSA} \geq 0.75$ to $<1.2 \mathrm{~m}^{2}: 800 \mathrm{mg} 3$ times daily with meals; titrate as needed by $400 \mathrm{mg}$

- phosphorus-free IV fluids

$\mathrm{mg} / \mathrm{mL}$ ) [>2.1 $\mathrm{mmol} / \mathrm{L}]$ )

per dose at 2 week intervals. 
Contd...

\begin{tabular}{lll}
\hline Clinical problem & Approach & Dosages \\
\hline Calcium car- & BSA $\geq 1.2 \mathrm{~m}^{2}: 1,600 \mathrm{mg}, 3$ times daily with \\
bonate & meals; titrate as needed by $800 \mathrm{mg}$ per dose \\
& at 2 weeks interval should be limited to \\
& $1,500 \mathrm{mg} /$ day of elemental calcium and total \\
& calcium intake (including dietary sources and \\
& calcium-based phosphate binders) should not \\
& exceed 2,000 mg/day of elemental calcium \\
& $0.5 \mathrm{~g} / \mathrm{kg}$ over 30 minutes \\
& Bextrose $(50 \%)$ \\
&
\end{tabular}

Comments and recommendations

- Phosphate binders may interfere with drug absorption If no response to medical therapy

Calcium gluconate $(10 \%)$

IV $100 \mathrm{mg} / \mathrm{kg}$ (maximum dose: 3,000
repeat in 10 minutes if necessary

Hyperkalemia

Hypocalcemia

Sodium polystyrene sulfonate (Kayexalate) maximum dose: $15 \mathrm{~g} /$ dose

Albuterol Inhaled $2 \mathrm{~mL}$ of saline $\mathrm{mL}$ of saline $\mathrm{mg}$ in $2-4 \mathrm{~mL}$ of saline

Calcium gluco-
Insulin (regular) 0.1 units per kg maximum dose of 10 units

Sodium bicar- $\quad 1 \mathrm{mEq} / \mathrm{kg}$ (maximum dose $50 \mathrm{mEq}$ ) IV over bonate 10-15 minutes
- Monitor glucose level regularly

- Time to onset of action is immediate

- Children younger than 5 years of age - give $10 \%$ dextrose $(100 \mathrm{mg} / \mathrm{mL})$ at a dose of 5 $\mathrm{mL} / \mathrm{kg}$

- Children 5 years of age and older - give $25 \%$ dextrose $(250 \mathrm{mg} / \mathrm{mL})$ at a dose of $2 \mathrm{~mL} / \mathrm{kg}$ (maximum dose $25 \mathrm{~g}$ )

- Rapid infusion may cause bradycardia

- In the clinical setting of a cardiac arrest or impending arrest, calcium chloride is typically used rather than calcium gluconate because it results in a more rapid increase in the serum ionized calcium

- At dose $20 \mathrm{mg} / \mathrm{kg}$ (maximum dose $1,000 \mathrm{mg}$ ) given over 5-10 minutes

- To be given along with dextrose infusion to avoid hypoglycemia

- Avoid giving at in the same line of calcium as it will block the line if no good flush

- It can be given as $1 \mathrm{~mL} / \mathrm{kg}$ of an $8.4 \%$ solution

- For children less than 6 months of age, as 2 $\mathrm{mL} / \mathrm{kg}$ of a $4.2 \%$ solution. Although dosing can be repeated, there is a risk of developing hypernatremia with repeated doses

- Oral, nasogastric: $1 \mathrm{~g} / \mathrm{kg} /$ dose every 6 hours; Oral route more effective than rectal

- Rectal: $1 \mathrm{~g} / \mathrm{kg} /$ dose every 2-6 hours maximum dose range: $30-50 \mathrm{~g} /$ dose

- Neonates $-0.4 \mathrm{mg}$ in $2 \mathrm{~mL}$ of saline

- Infants and small children $<25 \mathrm{~kg} 2.5 \mathrm{mg}$ in

In case of rectal administration retain at least 15-60 minutes

- Inhaled albuterol may also be administered by metered dose inhaler as 4-8 puffs with a spacer.

Can be given concomitantly with the combination of IV insulin and glucose, or sequentially for patients who may not respond adequately to insulin/glucose therapy.

- Children between 25 and $50 \mathrm{~kg} 5 \mathrm{mg}$ in 2 .

- Older children and adolescents $>50 \mathrm{~kg} 10$ • nate $(10 \%)$
IV $100 \mathrm{mg} / \mathrm{kg}$ (maximum dose: 3,000 mg) may . repeat in 10 minutes if necessary
Only if symptomatic; repeat as necessary; use with caution in patients with severe hyperphosphatemia
The good outcome of NIV for such patients has been reported in multiple published articles, for example, the retrospective studies by Schiller et al. which reported avoidance of intubation in patients received NIV via a full-face mask or a helmet. ${ }^{28-32}$
Considering the benefits of early NIV in adults' studies, it is important for the pediatric intensivist to consider a trial of NIV in these patients. However, there will be always situations where patients will require invasive ventilation depending on clinical 
severity and progression of ARDS. Close monitoring is essential, and timely switch to conventional invasive ventilation via endotracheal tube should be considered if there is no improvement within 2 hours of NIV.

The criteria for NIV failure in the PICU population are involvement of second organ failure, acidotic pH less than 7.25 after 2 hours of NIV, the need for a higher setting of support (mean pressure more than $12 \mathrm{~cm} \mathrm{H}_{2} \mathrm{O}$ or $\mathrm{FiO}_{2}$ more than $60 \%$ ), and the progressive changes on chest X-ray in clinical status of the patient. $^{33-35}$

\section{Cancer and Sepsis}

Several factors increase the risk of developing sepsis, which include the type of malignancy, level of treatment, and chemotherapy regimen being applied (some drugs are more myelosuppressive than others), duration and depth of neutropenia, and the integrity of skin and mucosal barriers. The presence of comorbidities and indwelling catheters also increases the risk of developing sepsis. ${ }^{36,37}$

The Sepsis-3 definitions call for a new clinical tool to replace the criteria for systemic inflammatory response syndrome (SIRS) in identifying patients with sepsis. As SIRS definition is nonclinically specific, and as criteria of sepsis does not accurately reflect the host-body response. Most cases of neutropenic fever are managed outside the PICU with broad-spectrum antibiotics. A pediatric intensivist is only involved in cases with sever sepsis or septic shock. It is useful to remember that the early warning sign such as fever is often absent in oncology patients.

Physicians therefore must rely on other parameters to determine sepsis, evidence of fluid redistribution, tachycardia, poor perfusion, and hypotension are all signs of impending and current sepsis. High serum lactate raised inflammatory markers (C-reactive protein and procalcitonin) or organs dysfunction aid the diagnosis and used as orientation in the treatment approach.

The cornerstone of sepsis treatment remains the empirical use of broad-spectrum antibiotic therapy. Multiple treatment protocols including both monotherapeutic and multidrug regimens have been documented to be effective, although no superiority for one regimen over another. Changes in the epidemiology of infectious organisms and the appearance of multidrug-resistant strains require from clinician continuous reevaluation of the antibiotic selection. ${ }^{38,39}$ Evidence-based guidelines for the management of febrile neutropenia have been developed by multiple globally well-known institutions. In practice, patients should receive the first dose of recommended wide spectrum gram-negative coverage antibiotic in the floor or emergency department before their admission to PICU.

Subsequent antibiotic management is directed by cultures. These patients are also at risk of contracting invasive fungal infections like Candida species or invasive aspergillosis, especially those with prolonged neutropenia and/or hematopoietic stem-cell transplant recipients. The best outcome in the treatment given by physician to patients with infectious disease is warranted. ${ }^{40-44}$ In supportive measures, granulocyte colony-stimulating factor administration should be a part of the treatment protocol for prolonged (more than 10 days) or profound severe neutropenia (ANC) less than 100, or in cases of uncontrolled primary oncological disease or multiorgan dysfunction syndrome. ${ }^{45}$

\section{Cancer and Cytokine Release Syndrome}

New novel immunotherapies such as chimeric antigen receptor T-cell therapies have recently been approved for clinical practice

after showing impressive therapeutic results in the treatment of refractory acute lymphoblastic B-cell leukemia. As such therapy protocols will increasingly be used in clinical practice plus the fact of reported severe inflammatory response and cytokine release syndrome (CRS) generated after its use as reported by Foster and Maude in 2018, it is very important that practising oncologists and intensive care specialists are familiar with the complications of such therapy and the treatment protocols (Table 2).

The pathophysiology of observed signs and symptoms is poorly understood and still, under investigation, interleukin-6 (IL-6) holds a key role in CRS pathophysiology. ${ }^{46-48}$ Treatment requires collaboration between oncologist and intensive care specialist without losing hope as even severe CRS has a relatively good prognosis when appropriately treated, patients with CRS should be offered the full support they need if necessary.

Symptomatic treatment with antihistamines, antipyretics, and fluids can be the start in mild cases. While not enough in other cases where the use of therapy directed toward key factors of the pathophysiology of CRS is warranted in the form of immunomodulation agents such as tocilizumab, by binding to membrane bound as well as soluble IL-6 receptor, tocilizumab interferes with both classical and trans-signaling pathways. Subsequent studies confirmed that the administration of monoclonal antibodies against IL-6 (siltuximab) and its receptor (tocilizumab) led to rapid resolution of CRS symptoms. ${ }^{25,49-51}$

Table 2: Effect of cytokine release syndrome on organs and systems $\mathrm{s}^{22-25}$

\begin{tabular}{ll}
\hline System involved & Observed signs and symptoms reported \\
\hline General appearance & $\begin{array}{l}\text { Mild symptoms of fever, fatigue, arthralgia, } \\
\text { and myalgia can be the start of } \\
\text { CRS storm. }\end{array}$
\end{tabular}

Hemodynamics and cardiovascular system

Respiratory system

Central nervous system

\section{Gastrointestinal} system

Kidneys and urinary tract

Blood and metabolic abnormalities
Tachycardia, hypotension, and septic shock with the need of vasopressors and inotropic support in ICUs have been reported also high level of troponin and cardiac arrhythmias in form of prolong Q-T interval after treatment, cardiomyopathies and acute heart failure observed in some cases.

Cough and tachypnea but can progress to acute respiratory distress syndrome (ARDS) with dyspnea, hypoxemia, may pulmonary edema and respiratory failure.

Neurotoxicity has been reported with wide range of clinical presentation from mild confusion with word-finding difficulty, headaches and hallucinations to aphasia, hemiparesis, cranial nerve palsies, seizures, and sever encephalopathies.

Sever acute mucosities, diarrhea, and colitis

Nausea and vomiting

Splenomegaly, hepatomegaly, elevation of liver enzymes, liver failure

Acute kidney injury and hematuria

Cytopenias, coagulopathy, disseminated intravascular coagulation

High CRP, elevated ferritin levels, and hypertriglyceridemia 


\section{Cancer and Nervous System}

Any part of the nervous system can be affected. Neurological presentations also vary significantly depending upon the location, size, and biological behavior of the tumor. Presenting symptoms of CNS tumors include headache, vomiting, altered consciousness, gait disturbance, cranial nerve palsies, long tract signs and behavior changes, seizures, growth, and endocrine disturbances among others.

Leukemic infiltration of CNS can vary in presentation from asymptomatic to seizures and deposition of solid masses of leukemic blasts.

Chemotherapy treatment side effects can present with signs of raised intracranial pressure, posterior reversible encephalopathy syndrome, and leukoencephalopathy secondary to chemotherapeutic agents. Spinal tumors often present in the form of back pain or paraplegia. There are often subtle symptoms predating the catastrophic signs of cord compression.

Most CNS manifestations are observed and treated by the neurology and oncology teams. Only a small proportion of these children are admitted to the PICU at some point during their treatment due to:

- Postoperative monitoring after tumor resection or biopsy, external ventricular drainage, or ventriculoperitoneal shunt insertions,

- Uncontrolled seizures/status epilepticus,

- Complications caused by increased pressure in the brain and obstructive hydrocephalus,

- Ischemic stroke, hemorrhage, and

- Spinal cord compression.

The treatment of neurological presentations/complications is directed toward treating the primary cause. This involves directly neurosurgical intervention or anticancer treatment in the form of chemotherapy or radiotherapy.

\section{Anticancer Therapy Requiring PICU Support}

A lot of chemotherapy used in practice and it is important to know its side effects to predict complications and to direct treatment accordingly (Table 3).

\section{Conclusion}

Following the recent updates in the treatment protocols, pediatric oncology requires awareness from PICU doctors about common emergencies and life-threatening complications that can happen during any stage of this illness.

Even PICU admission criteria and treatment considerations for such patients are different from the general PICU patients, which highlights the importance of keeping up to date with the last recommendation and treatment approaches without forgetting the importance of a multidisciplinary approach side by side with the pediatric oncologist to achieve best treatment and outcome.

\section{References}

1. Von bergwelt-Baildon $M$, Hallek MJ, Shimabukuro-Vornhagen AA, Kochanek M. CCC meets ICU: redefining the role of critical care of cancer patients. BMC Cancer 2010;10(1):612. DOI: 10.1186/1471-240710-612. http://www.biomedcentral.com/1471-2407/10/612.

2. Brunet F, Lanore JJ, Dhainaut JF, Dreyfus F, Vaxelaire JF, Nouira S, et al. Is intensive care justified for patients with haematological
Table 3: Anticancer agents with indications for PICU involvement

\begin{tabular}{ll}
\hline Anticancer agents & Indications for PICU involvement \\
\hline L-Asparaginase & Acute pancreatitis \\
L-Asparaginase & Allergic reaction
\end{tabular}

Rituximab, alemtuzumab, gem-

tuzumab

Antithymocyte globulin, gamma

globulin

Etoposide, carboplatin

Blood products (especially in

post-stemcell transplant

setting)

Antimetabolites (cytarabine,

fludarabine, capecitabine)

Alkylating agents (busulfan, cyclophosphamide, ifosfamide)

Anthracyclines (daunorubicin, doxorubicin, idarubicin, mitoxantrone)

Anthracyclines, cyclophosphamide, ifosfamide

Rituximab, alemtuzumab, tisagenlecleucel, cytarabine

Methotrexate, ifosfamide

Cyclophosphamide, ifosfamide, radiotherapy

All trans-retinoic acid

Actinomycin-D, busulfan, cyclophosphamide, cytarabine, fludarabine, gemtuzumab, mercaptopurine, roxithromycin, thioguanine, vincristine

malignancies? Intensive Care Med 1990;16(5):291-297. DOI: 10.1007/ BF01706352.

3. Carlon GC. Admitting cancer patients to the intensive care unit. Crit Care Clin 1988;4(1):183-191. DOI: 10.1016/S0749-0704(18)30511-6.

4. Garrouste-Orgeas M, Boumendil A, Pateron D, Aergerter P, Somme $D$, Simon T, et al. Selection of intensive care unit admission criteria for patients aged 80 years and over and compliance of emergency and intensive care unit physicians with the selected criteria: an observational multicenter, prospective study. Crit Care Med 2009;37(11):2919-2928. DOI: 10.1097/CCM.0b013e3181b019f0.

5. Dalton HJ, Slonim AD, Pollack MM. MultiCenter outcome of pediatric oncology patients requiring intensive care. Pediatr Hematol Oncol 2003;20(8):643-649. DOI: 10.1080/08880010390243095.

6. Gullberg N, Kalzén H, Luhr O, Göthberg S, Winsö O, Markström A, et al. Immediate and 5-year cumulative outcome after paediatric intensive care in Sweden. Acta Anaesthesiol Scand 2008;52(8):1086-1095. DOI: 10.1111/j.1399-6576.2008.01711.x.

7. Haase R, Mathony U, Lieser U, Nagel F, Sitka U, Burdach S. Oncology patients in a pediatric intensive care unit-a 7-year experience. Klin Padiatr 2003;215(4):234-240. DOI: 10.1055/s-2003-41399.

8. Owens C, Mannion D, O'Marcaigh A, Waldron M, Butler K, O'Meara A. Indications for admission, treatment and improved outcome of paediatric haematology/oncology patients admitted to a tertiary paediatric ICU. Ir J Med Sci 2011;180(1):85-89. DOI: 10.1007/s11845010-0634-8.

9. Hallahan AR, Shaw PJ, Rowell G, O'Connell A, Schell D, Gillis J. Improved outcomes of children with malignancy admitted to a 
pediatric intensive care unit. Crit Care Med 2000;28(11):3718-3721. DOI: 10.1097/00003246-200011000-00030.

10. Heying R, Schneider DT, Körholz D, Stannigel H, Lemburg P, Göbel U. Efficacy and outcome of intensive care in pediatric oncologic patients. Crit Care Med 2001;29(12):2276-2280. DOI: 10.1097/00003246200112000-00007.

11. Dursun O, Hazar V, Karasu GT, Uygun V, Tosun O, Yesilipek A. Prognostic factors in pediatric cancer patients admitted to the pediatric intensive care unit. J Pediatr Hematol Oncol 2009;31(7):481-484. DOI: 10.1097/ MPH.0b013e3181a330ef

12. Meyer S, Gottschling S, Biran T, Georg T, Ehlayil K, Graf N, et al. Assessing the risk of mortality in paediatric cancer patients admitted to the paediatric intensive care unit: a novel risk score? Eur J Pediatr 2005;164(9):563-567. DOI: 10.1007/s00431-005-1695-y.

13. Stucki A, Rivier AS, Gikic M, Monai N, Schapira M, Spertini O Endothelial cell activation by myeloblasts: molecular mechanisms of leukostasis and leukemic cell dissemination. Blood 2001;97(7):2121. DOI: 10.1182/blood.V97.7.2121.

14. Azoulay É, Fieux F, Moreau D, Thiery G, Rousselot P, Parrot A, et al. Acute monocytic leukemia presenting as acute respiratory failure. Am J Respir Crit Care Med 2003;167(10):1329-1333. DOI: 10.1164/ rccm.200206-554OC

15. Bug G, Anargyrou K, Tonn T, Bialleck H, Seifried E, Hoelzer D, et al. Impact of leukapheresis on early death rate in adult acute myeloid leukemia presenting with hyperleukocytosis. Transfusion 2007:47(10):1843. DOI: 10.1111/j.1537-2995.2007.01406.x.

16. Wilson L, Detterbeck F, Yahalom J. Superior vena cava syndrome with malignant causes. N Engl J Med 2007;356(18):1862-1869. DOI: 10.1056/NEJMcp067190.

17. National Institute for Health and Care Excellence, 2008. Metastatic spinal cord compression: diagnosis and management of patients at risk of or with metastatic spinal cord compression. (CG75.) www. nice. org.uk/guidance/CG75/ [Accessed 5 August 2014].

18. Loblaw DA, Laperriere NJ, Mackillop WJ. A population-based study of malignant spinal cord compression in Ontario. Clin Oncol 2003;15(4):211-217. DOI: 10.1016/S0936-6555(02)00400-4.

19. Howard SC, Ribeiro RC, Pui C-H. Acute complications Pui C-H, ed. Childhood leukemias. Cambridge, United Kingdom: Cambridge University Press; 2006. pp. 709-749.

20. Alakel N, Middeke JM, Schetelig J, Bornhäuser M. Prevention and treatment of tumor lysis syndrome, and the efficacy and role of rasburicase. Onco Targets Ther 2017;10:597-605. DOI: 10.2147/OTT. S103864.

21. Halfdanarson TR, Hogan WJ, Moynihan TJ. Oncologic emergencies: diagnosis and treatment. Mayo Clin Proc 2006;81(6):835-848. DOI: 10.4065/81.6.835

22. Hay KA, Hanafi L-A, Li D, Gust J, Liles WC, Wurfel MM, et al. Kinetics and biomarkers of severe cytokine release syndrome after CD19 chimeric antigen receptor-modified T cell therapy. Blood 2017;130(21):22952306. DOI: 10.1182/blood-2017-06-793141.

23. Kantarjian H, Stein A, Gökbuget N, Fielding AK, Schuh A, Ribera $J M$, et al. Blinatumomab versus chemotherapy for advanced acute lymphoblastic leukemia. N Engl J Med 2017;376(9):836-847. DOI: 10.1056/NEJMoa1609783.

24. Neelapu SS, Tummala S, Kebriaei P, Wierda W, Gutierrez C, Locke $\mathrm{FL}$, et al. Chimeric antigen receptor T-cell therapy - assessment and management of toxicities. Nat Rev Clin Oncol 2018;15(1):47-62. DOI: 10.1038/nrclinonc.2017.148.

25. Lee DW, Gardner R, Porter DL, Louis CU, Ahmed N, Jensen M, et al. Current concepts in the diagnosis and management of cytokine release syndrome. Blood 2014;124(2):188-195. DOI: 10.1182/blood2014-05-552729.

26. Bernard GR, Artigas A, Brigham KL, Carlet J, Falke K, Hudson L, et al. The American-European consensus conference on ARDS. Definitions, mechanisms, relevant outcomes, and clinical trial coordination. Am J Respir Crit Care Med 1994;149(3):818-824. DOI: 10.1164/ ajrccm.149.3.7509706.
27. Khemani RG, Thomas NJ, Venkatachalam V, Scimeme JP, Berutti T, Schneider JB, et al. Comparison of $\mathrm{SpO} 2$ to $\mathrm{PaO} 2$ based markers of lung disease severity for children with acute lung injury*. Crit Care Med 2012;40(4):1309-1316. DOI: 10.1097/CCM.0b013e31823bc61b.

28. Squadrone $V$, Massaia $M$, Bruno $B$, Marmont $F$, Falda $M$, Bagna $C$, et al. Early CPAP prevents evolution of acute lung injury in patients with hematologic malignancy. Intensive Care Med 2010;36(10):1666-1674. DOI: 10.1007/s00134-010-1934-1.

29. Azoulay E, Alberti C, Bornstain C, Leleu G, Moreau D, Recher C, et al. Improved survival in cancer patients requiring mechanical ventilatory support: impact of noninvasive mechanical ventilatory support. Crit Care Med 2001;29(3):519-525. DOI: 10.1097/00003246-20010300000009.

30. Antonelli M, Conti G, Bufi M, Costa MG, Lappa A, Rocco M, et al. Noninvasive ventilation for treatment of acute respiratory failure in patients undergoing solid organ transplantation: a randomized trial. JAMA 2000;283(2):235-241. DOI: 10.1001/jama.283.2.235.

31. Hilbert G, Gruson D, Vargas F, Valentino R, Gbikpi-Benissan G, Dupon $M$, et al. Noninvasive ventilation in immunosuppressed patients with pulmonary infiltrates, fever, and acute respiratory failure. $\mathrm{N}$ Engl J Med 2001;344(7):481-487. DOI: 10.1056/NEJM200102153440703.

32. Pancera CF, Hayashi M, Fregnani JH, Negri EM, Deheinzelin D, de Camargo B. Noninvasive ventilation in immunocompromised pediatric patients: eight years of experience in a pediatric oncology intensive care unit. J Pediatr Hematol Oncol 2008;30(7):533-538. DOI: 10.1097/MPH.0b013e3181754198.

33. Dohna-Schwake C, Stehling F, Tschiedel E, Wallot M, Mellies U. Noninvasive ventilation on a pediatric intensive care unit: feasibility, efficacy, and predictors of success. Pediatr Pulmonol 2011;46(11):11141120. DOI: 10.1002/ppul.21482.

34. Muñoz-Bonet Jl,Flor-Macián EM, Brines J, Roselló-Millet PM, Llopis MC, López-Prats JL, et al. Predictive factors for the outcome of noninvasive ventilation in pediatric acute respiratory failure. Pediatr Crit Care Med 2010;11(6):675-680. DOI: 10.1097/PCC.0b013e3181d8e303.

35. Essouri S, Chevret L, Durand P, Haas V, Fauroux B, Devictor D. Noninvasive positive pressure ventilation: five years of experience in a pediatric intensive care unit. Pediatr Crit Care Med 2006;7(4):329-334. DOI: 10.1097/01.PCC.0000225089.21176.0B

36. Pound CM, Johnston DL, Armstrong R, Gaboury I, Menon K. The morbidity and mortality of pediatric oncology patients presenting to the intensive care unit with septic shock. Pediatr Blood Cancer 2008;51(5):584-588. DOI: 10.1002/pbc.21670.

37. Tamburro R. Pediatric cancer patients in clinical trials of sepsis: factors that predispose to sepsis and stratify outcome. Pediatr Crit Care Med 2005;6(Supplement):S87-S91. DOI: 10.1097/01. PCC.0000161288.00396.49.

38. Goldstein B, Giroir B, Randolph A. International pediatric sepsis consensus conference: definitions for sepsis and organ dysfunction in pediatrics. Pediatr Crit Care Med 2005;6(1):2-8. DOI: 10.1097/01. PCC.0000149131.72248.E6.

39. James W. Hathorn and Kirsten Lyke. Clin Infect Dis 1997;24(Supplement_2):S256-S265. DOI: https://doi.org/10.1093/ clinids/24.Supplement_2.S256.

40. Klastersky J, de Naurois J, Rolston K, Rapoport B, Maschmeyer G, Aapro M, et al. Management of febrile neutropaenia: ESMO clinical practice guidelines. Ann Oncol 2016;27(suppl 5):v111-v118. DOI: 10.1093/annonc/mdw325.

41. Neutropenia and risk of infection. CDC website. www.cdc.gov/cancer/ preventinfections/pdf/neutropenia.pdf. Accessed August 2, 2017.

42. Vanderpuye-Orgle J, Sexton Ward A, Huber C, Kamson C, Jena AB. Estimating the social value of G-CSF therapies in the United States. Am J Manag Care 2016;22(10):e343-e349.

43. Freifeld AG, Bow EJ, Sepkowitz KA, Boeckh MJ, Ito Jl, Mullen CA, et al., Infectious Diseases Society of America. Clinical practice guideline for the use of antimicrobial agents in neutropenic patients with cancer: 2010 update by the Infectious Diseases Society of America. Clin Infect Dis 2011;52(4):e56-e93. DOI: 10.1093/cid/cir073. 
44. NCCN Clinical Practice Guidelines in Oncology. Prevention and Treatment of Cancer-Related Infections, version 2. 2017. National Cancer Comprehensive Network website. www.nccn.org/ professionals/physician_gls/PDF/infections.pdf. Published February 21, 2017. Accessed September 27, 2017. 10.4049/jimmunol.1500861.

45. Smith TJ, Khatcheressian J, Lyman GH, Ozer H, Armitage JO, Balducci $L$, et al. Update of recommendations for the use of white blood cell growth factors: an evidence-based clinical practice guideline. J Clin Oncol 2006;24(19):3187-3205. DOI: 10.1200/JCO.2006.06.4451.

46. Teachey DT, Rheingold SR, Maude SL, Zugmaier G, Barrett DM, Seif $A E$, et al. Cytokine release syndrome after blinatumomab treatment related to abnormal macrophage activation and ameliorated with cytokine-directed therapy. Blood 2013;121(26):5154-5157. DOI: 10.1182/blood-2013-02-485623.

47. Brentjens RJ, Davila ML, Riviere I, Park J, Wang X, Cowell LG, et al. CD19-targeted $T$ cells rapidly induce molecular remissions in adults with chemotherapy-refractory acute lymphoblastic leukemia. Sci Transl Med 2013;5(177):177ra38. DOI: 10.1126/scitransImed.3005930.

48. Tanaka T, Narazaki M, Kishimoto T. Immunotherapeutic implications of IL-6 blockade for cytokine storm. Immunotherapy 2016;8(8):959-970. DOI: 10.2217/imt-2016-0020.

49. Brudno JN, Kochenderfer JN. Toxicities of chimeric antigen receptor T cells: recognition and management. Blood 2016;127(26):3321-3330. DOI: 10.1182/blood-2016-04-703751.

50. Hu Y, Wu Z, Luo Y, Shi J, Yu J, Pu C, et al. Potent anti-leukemia activities of chimeric antigen receptor-modified TCells against CD19 in Chinese patients with relapsed/refractory acute lymphocytic leukemia. Clin Cancer Res 2017;23(13):3297-3306. DOI: 10.1158/1078-0432.CCR-161799.

51. Bonifant $\mathrm{CL}$, Jackson HJ, Brentjens RJ, Curran KJ. Toxicity and management in CART-cell therapy. Mol Ther Oncolytics 2016;3:16011. DOI: $10.1038 /$ mto.2016.11 